University of New Orleans

ScholarWorks@UNO

5-1-1999

\title{
Performance optimization and light-beam-deviation analysis of the parallel-slab division-of-amplitude photopolarimeter
}

Aed M. El-Saba

Rasheed M.A. Azzam

University of New Orleans, razzam@uno.edu

Mustafa A. G. Abushagur

Follow this and additional works at: https://scholarworks.uno.edu/ee_facpubs

Part of the Electrical and Electronics Commons

\section{Recommended Citation}

Aed M. El-Saba, Rasheed M. A. Azzam, and Mustafa A. G. Abushagur, "Performance Optimization and Light-Beam-Deviation Analysis of the Parallel-Slab Division-of-Amplitude Photopolarimeter," Appl. Opt. 38, 2829-2836 (1999)

This Article is brought to you for free and open access by the Department of Electrical Engineering at ScholarWorks@UNO. It has been accepted for inclusion in Electrical Engineering Faculty Publications by an authorized administrator of ScholarWorks@UNO. For more information, please contact scholarworks@uno.edu. 


\title{
Performance optimization and light-beam-deviation analysis of the parallel-slab division-of-amplitude photopolarimeter
}

\author{
Aed M. El-Saba, Rasheed M. A. Azzam, and Mustafa A. G. Abushagur
}

\begin{abstract}
A division-of-amplitude photopolarimeter that uses a parallel-slab multiple-reflection beam splitter was described recently [Opt. Lett. 21, 1709 (1996)]. We provide a general analysis and an optimization of a specific design that uses a fused-silica slab that is uniformly coated with a transparent thin film of $\mathrm{ZnS}$ on the front surface and with an opaque $\mathrm{Ag}$ or Au reflecting layer on the back. Multiple internal reflections within the slab give rise to a set of parallel, equispaced, reflected beams numbered $0,1,2$, and 3 that are intercepted by photodetectors $D_{0}, D_{1}, D_{2}$, and $D_{3}$, respectively, to produce output electrical signals $i_{0}, i_{1}, i_{2}$, and $i_{3}$, respectively. The instrument matrix $\mathbf{A}$, which relates the output-signal vector I to the input Stokes vector $\mathbf{S}$ by $\mathbf{I}=\mathbf{A S}$, and its determinant $D$ are analyzed. The instrument matrix A is nonsingular; hence all four Stokes parameters can be measured simultaneously over a broad spectral range (UV-VIS-IR). The optimum film thickness, the optimum angle of incidence, and the effect of light-beam deviation on the measured input Stokes parameters are considered. (C) 1999 Optical Society of America

OCIS codes: $120.5700,220.2740,310.1620$.
\end{abstract}

\section{Introduction}

Fast measurement of the complete state of polarization (SOP) of light, as determined by the four Stokes parameters, requires systems that employ no moving parts or modulators. This constraint has prompted the development of new, simple, and rugged photopolarimeters that operate without moving parts or modulators. ${ }^{1-4}$ One class of such instruments uses division of the wave front, ${ }^{5-7}$ whereas another uses division of amplitude..$^{8-11}$ In the latter class the input light beam whose SOP is to be measured is divided into four or more beams that are intercepted by discrete (or array) photodetectors. Each detector $D_{k}(k=0,1,2$, 3) generates an electrical signal $i_{k}(k=0,1,2,3)$ proportional to the fraction of the radiation it absorbs. Linear detection of the light fluxes of the four component beams determines the four Stokes parameters of

A. M. El-Saba (ame@ece.uah.edu) and M. A. G. Abushagur are with the Department of Electrical and Computer Engineering, University of Alabama at Huntsville, Huntsville, Alabama 35899. R. M. A. Azzam is with the Department of Electrical Engineering, University of New Orleans, New Orleans, Louisiana 70148.

Received 22 October 1998; revised manuscript received 17 February 1999.

0003-6935/99/132829-08\$15.00/0

(C) 1999 Optical Society of America the incident light by means of an instrument matrix (IM) A that is obtained by calibration.

In the parallel-slab (PS) division-of-amplitude photopolarimeter (DOAP), or the PS-DOAP, a parallelplane dielectric slab of refractive index $N_{1}(\lambda)$ and thickness $d$ replaces the three beam splitters of the DOAP. Figure 1 shows the basic arrangement of the PS-DOAP. The bottom surface of the slab is coated with an opaque, highly reflective metal of complex refractive index $N_{2}(\lambda)=n_{2}-j k_{2}$, where $\lambda$ is the wavelength of light. The light beam whose SOP is to be measured is incident from air or vacuum $\left(N_{0}=1\right)$ upon the top surface of the slab (which may be bare or coated) at an angle $\phi_{0}$. Multiple internal reflections within the slab give rise to a set of parallel, equispaced, reflected beams (numbered $0,1,2,3, \ldots$ ) that are intercepted by photodetectors $\left(D_{0}, D_{1}, D_{2}, D_{3}, \ldots\right.$, respectively) to produce output electrical signals $\left(i_{0}, i_{1}\right.$, $i_{2}, i_{3}, \ldots$, respectively). Linear polarizers (or analyzers) $\left(A_{0}, A_{1}, A_{2}, A_{3}, \ldots\right)$ are placed in the respective reflected beams between the slab and the detectors. The insertion of these linear polarizers in front of the detectors has been noted to increase the polarization sensitivity greatly. ${ }^{12}$ The transmission axes of these polarizers are inclined with respect to the plane of incidence, which is the plane of the page in Fig. 1, by azimuth angles $\left(\alpha_{0}, \alpha_{1}, \alpha_{2}, \alpha_{3}, \ldots\right.$, respectively) that are measured in a counterclockwise (positive) sense 
looking toward the source. With linear detection the output signal of the $k$ th detector is a linear combination of the four Stokes parameters $S_{k}(k=0,1,2,3)$ of the incident light, i.e.,

$$
i_{k}=\sum_{k=0}^{3} a_{m k} S_{k}, \quad m=0,1,2,3, \ldots
$$

The $k$ th projection vector $\mathbf{a}_{k}=\left[a_{k 0} a_{k 1} a_{k 2} a_{k 3}\right]$ is equal to the first row of the Mueller matrix of the $k$ th light path from the source to the detector. When four signals are detected the output-current vector $\mathbf{I}=\left[i_{0} i_{1} i_{2}\right.$ $\left.i_{3}\right]^{t}$ (where $t$ stands for transpose) is linearly related to the input Stokes vector $\mathbf{S}=\left[S_{0} S_{1} S_{2} S_{3}\right]^{t}$ by

$$
\mathbf{I}=\mathbf{A S},
$$

where $\mathbf{A}$ is a $4 \times 4$ IM whose rows $\mathbf{a}_{k}$ are characteristic of the PS-DOAP at a given wavelength. The IM A is measured experimentally by calibration ${ }^{13,14}$; subsequently, the unknown incident Stokes vector $\mathbf{S}$ is obtained by

$$
\mathbf{S}=\mathbf{A}^{-1} \mathbf{I}
$$

\section{Determination of the Instrument Matrix of the} Parallel-Slab Division-of-Amplitude Photopolarimeter

The reflection Mueller matrix of the $k$ th order is given by ${ }^{15}$

$$
\begin{aligned}
& \left.-a_{11} a_{20}\right)+\left(a_{02} a_{23}-a_{03} a_{22}\right)\left(a_{11} a_{30}-a_{10} a_{31}\right) \\
& \left.+\left(a_{02} a_{13}-a_{03} a_{12}\right)\left(a_{20} a_{31}-a_{21} a_{30}\right)\right],
\end{aligned}
$$

where

$$
\begin{aligned}
& W_{1}=k_{0} k_{1} k_{2} k_{3}, \\
& W_{2}=R_{0} R_{1} R_{2} R_{3}, \\
& a_{k 0}=1-\cos 2 \alpha_{k} \cos 2 \psi_{k}, \\
& a_{k 1}=\cos 2 \alpha_{k}-\cos 2 \psi_{k}, \\
& a_{k 2}=\sin 2 \alpha_{k} \sin 2 \psi_{k} \cos \Delta_{k}, \\
& a_{k 3}=\sin 2 \alpha_{k} \sin 2 \psi_{k} \sin \Delta_{k}, \quad k=0,1,2,3 .
\end{aligned}
$$

For simplicity, we assume that the polarizers are oriented at uniformly distributed azimuths: $\alpha_{0}=90^{\circ}, \alpha_{1}=45^{\circ}, \alpha_{2}=0^{\circ}, \alpha_{3}=-45^{\circ}$. This assumption simplifies the IM considerably, and Eq. (6) becomes

$$
\begin{aligned}
D= & \left\{W_{1} W_{2} / 16\right\}\left\{\left(1+\cos 2 \psi_{0}\right)\left(1-\cos 2 \psi_{2}\right)\left(\sin 2 \psi_{1}\right)\right. \\
& \left.\times\left(\sin 2 \psi_{3}\right)\left[\sin \left(\Delta_{1}-\Delta_{3}\right)\right]\right\} .
\end{aligned}
$$

\section{Analysis of the Singularities of the Instrument Matrix of the Parallel-Slab Division-of-Amplitude Photopolarimeter}

$$
\mathbf{M}_{k}=R_{k}\left[\begin{array}{cccc}
1 & -\cos 2 \psi_{k} & 0 & 0 \\
\cline { 3 - 4 } \cos 2 \psi_{k} & 1 & 0 & 0 \\
0 & 0 & \sin 2 \psi_{k} \cos \Delta_{k} & \sin 2 \psi_{k} \sin \Delta_{k} \\
0 & 0 & -\sin 2 \psi_{k} \cos \Delta_{k} & \sin 2 \psi_{k} \cos \Delta_{k}
\end{array}\right]
$$

In Eq. (4), $\psi_{k}$ and $\Delta_{k}$ are the ellipsometric angles that characterize the interaction of the incident light beam with the slab that produces the $k$ th reflected order and $R_{k}$ is the power reflectance of the slab for the $k$ th reflected order for incident unpolarized light. The ideal polarizer (analyzer) matrix with an azimuth $\alpha_{k}$ is given by ${ }^{15}$

$$
\begin{aligned}
& \mathbf{P}_{k}= \\
& 1 / 2\left[\begin{array}{cccc}
1 & \cos 2 \alpha_{k} & \sin 2 \alpha_{k} & 0 \\
\cos 2 \alpha_{k} & \cos ^{2} 2 \alpha_{k} & \sin 2 \alpha_{k} \cos 2 \alpha_{k} & 0 \\
-\sin 2 \alpha_{k} & \sin 2 \alpha_{k} \cos 2 \alpha_{k} & \sin ^{2} 2 \alpha_{k} & 0 \\
0 & 0 & 0 & 0
\end{array}\right] .
\end{aligned}
$$

Carrying out an analysis similar to that for the grating DOAP ${ }^{12}$ reveals the general determinant of $\mathbf{A}$ to be

$$
\begin{aligned}
D= & \left\{W_{1} W_{2} / 16\right\}\left[\left(a_{00} a_{11}-a_{01} a_{10}\right)\left(a_{22} a_{33}-a_{23} a_{32}\right)\right. \\
& +\left(a_{00} a_{21}-a_{01} a_{20}\right)\left(a_{13} a_{32}-a_{12} a_{33}\right)+\left(a_{00} a_{31}\right. \\
& \left.-a_{01} a_{30}\right)\left(a_{12} a_{23}-a_{13} a_{22}\right)+\left(a_{02} a_{33}-a_{03} a_{32}\right)\left(a_{10} a_{21}\right.
\end{aligned}
$$

From Eq. (3) it is required that $\mathbf{A}^{-1}$ exist for the unambiguous determination of the full Stokes vector $\mathbf{S}$ from the output-current vector $\mathbf{I}$. This means that the IM A must be nonsingular and its determinant $D$ must be nonzero. From Eq. (8), we have $D=0$, and the IM A is singular if any of the multiplicative terms is zero. These singularities are grouped as follows:

1. $W_{1}=0$ : The responsivity of any detector is zero; the corresponding output signal disappears, and a measurement is lost.

2. $W_{2}=0$ : The power reflectance of the slab for any reflected order becomes zero.

3 . The zeroth order is purely $p$ polarized $\left(\psi_{0}=\right.$ $90^{\circ}$ ), so the slab functions as a linear polarizer in this order.

4. The second order is purely $s$ polarized $\left(\psi_{2}=0^{\circ}\right)$, so the slab functions as a linear polarizer in this order.

5. The $p$ or the $s$ polarization is suppressed in the first or the third order, i.e., $\psi_{1}$ or $\psi_{3}$ equals $0^{\circ}$ or $90^{\circ}$. This means that the slab functions as a linear polarizer in one of these orders. 


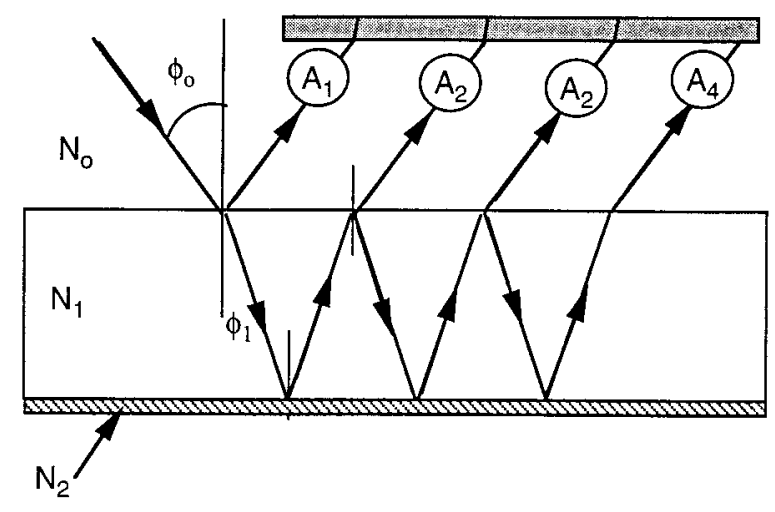

Fig. 1. Diagram of the PS-DOAP.

6. The differential-reflection phase shifts $\Delta_{1}$ and $\Delta_{3}$ of the first and the third orders, respectively, happen to be equal or differ by $\pm 180^{\circ}$.

To see whether one or more of these singularities can take place, let us consider a specific example of a fused-silica $\left(\mathrm{SiO}_{2}\right)$ dielectric slab that is coated on the back with Ag. At a wavelength of $633 \mathrm{~nm}$ the indices of refraction of $\mathrm{SiO}_{2}$ and $\mathrm{Ag}$ are taken (from Ref. 16) to be $N_{1}=1.456$ and $N_{2}=0.14-j 4.02$, respectively. Figure 2 shows the ellipsometric parameters $\psi_{k}[k=0,1,2,3$ (in degrees) $]$ for the entire range of $\phi_{0}$ for the first four reflected orders. Figure 2 indicates that, at $\phi_{0}=\phi_{B}$ (the Brewster angle of incidence), $\psi_{2}=\psi_{3}=0$; hence double-psi singularities exist at $\phi_{B}$.

Figure 3 shows the difference of the differential phase shifts $\left(\Delta_{1}-\Delta_{3}\right)$ between the second and the fourth reflected beams as a function of $\phi_{0}$. Figure 3 indicates that no delta singularities exist for any value of $\phi_{0}>0$.

Figure 4 shows a plot of the power reflectance $R_{k}$ $(k=0,1,2,3)$ of the slab for the first four reflected

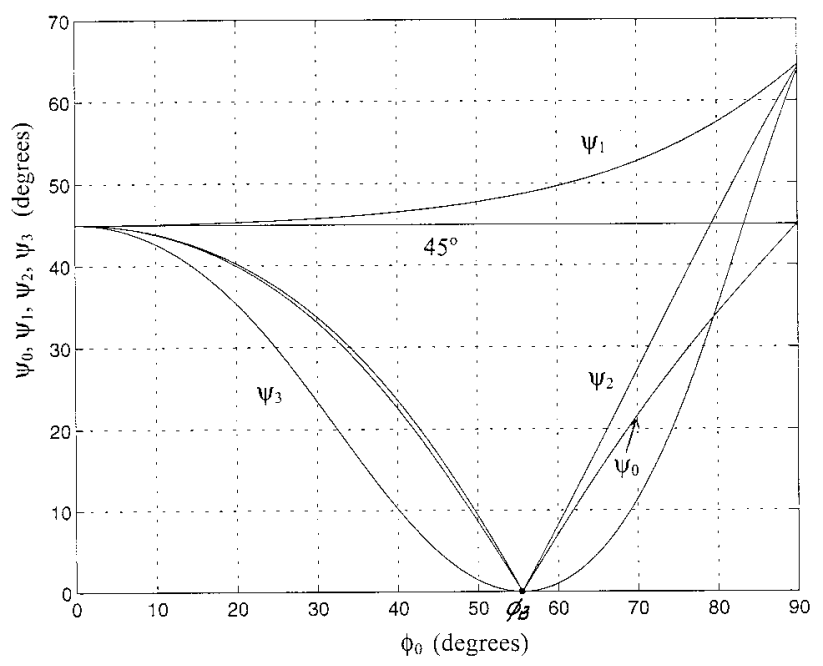

Fig. 2. Ellipsometric angle $\psi_{k}(k=0,1,2,3)$ for the first four reflected orders as functions of the incidence angle $\phi_{0}$ obtained by use of an uncoated $\mathrm{SiO}_{2}-\mathrm{Ag}$ parallel slab at $\lambda=633 \mathrm{~nm}$.

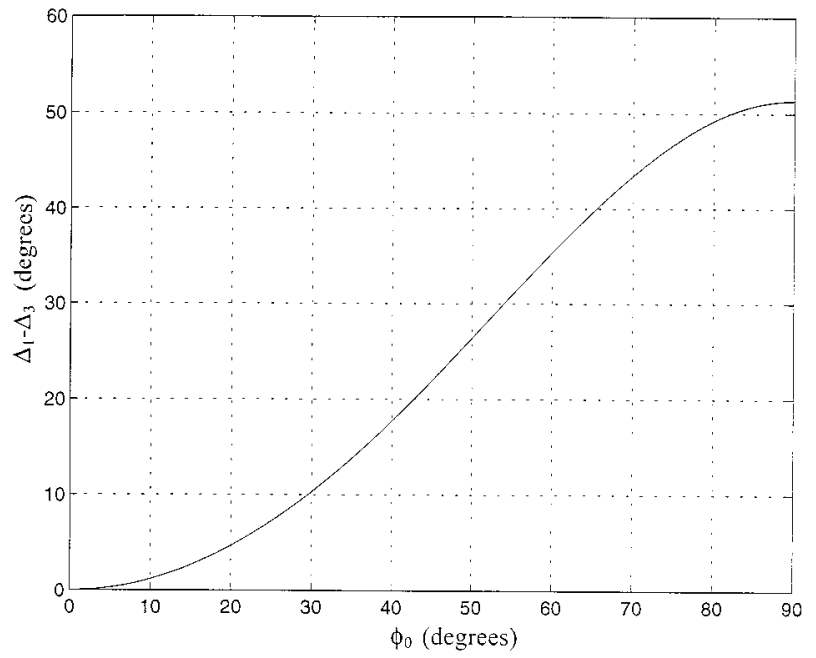

Fig. 3. Ellipsometric parameter $\Delta_{1}-\Delta_{3}$ as a function of the angle of incidence $\phi_{0}$ obtained by use of an uncoated $\mathrm{SiO}_{2}-\mathrm{Ag}$ parallel slab at $\lambda=633 \mathrm{~nm}$.

orders. We can see from Fig. 4 that $R_{3}$ is negligible for values of $\phi_{0}$ as great as $60^{\circ}$, which means that, for the fourth beam to have any significant power, the PS-DOAP has to operate at a high angle of incidence. In operating this system at $\phi_{0}<60^{\circ}, R_{3}$ is small, and a singularity essentially takes place, as was discussed above.

Figure 5 shows the normalized determinant $D_{N}$ of the IM [obtained by division of the right-hand side of Eq. (8) by $\left.W_{1} W_{2} / 16\right]$ plotted as a function of $\phi_{0}$. We emphasize that this is the normalized determinant and that any singularities owing to $W_{1}$ or $W_{2}$ will not show up in $D_{N}$. Figure 5 shows a flat singularity in the range $50^{\circ}<\phi_{0}<60^{\circ}$. The flatness of $D_{N}$ is due to the flatness of the singularity, $\psi_{3} \cong 0$, and to the double singularities of $\psi_{2}$ and $\psi_{3}$ for $50^{\circ}<\phi_{0}<60^{\circ}$. Figure 5 also suggests that optimum performance of this PS-

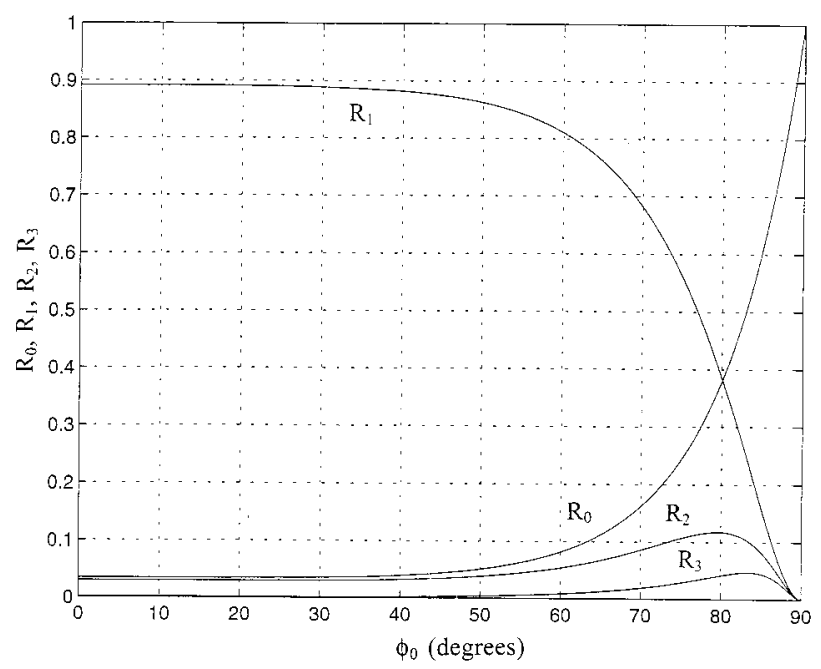

Fig. 4. Power reflectance $R_{k}(k=0,1,2,3)$ for the first four reflected orders as functions of the incidence angle $\phi_{0}$ obtained by use of an uncoated $\mathrm{SiO}_{2}-\mathrm{Ag}$ parallel slab at $\lambda=633 \mathrm{~nm}$. 


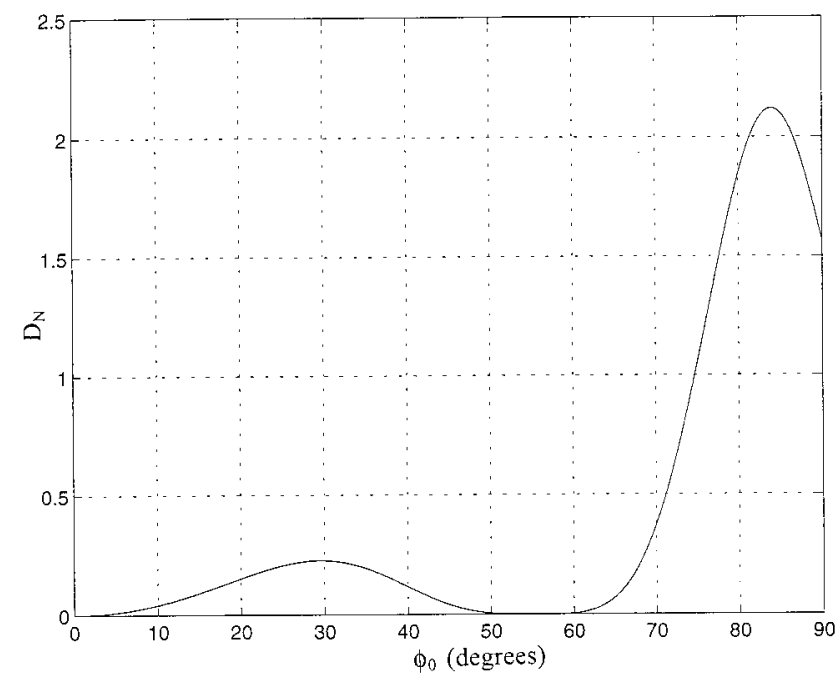

Fig. 5. Normalized determinant $D_{N}$ as a function of the incidence angle $\phi_{0}$ obtained by use of an uncoated $\mathrm{SiO}_{2}-\mathrm{Ag}$ parallel slab at $\lambda$ $=633 \mathrm{~nm}$.

DOAP occurs at $\phi_{\text {optm }} \cong 82^{\circ}$, where $D_{N}$ is its maximum $D_{N \max }$. However, operation of the PS-DOAP at $\phi_{0} \cong$ $82^{\circ}$ is impractical because of field-of-view restrictions. In Section 4 we show that performance can be improved by means of coating the top surface of the $\mathrm{SiO}_{2}$ slab with a thin film. Coating the top surface increases the power in the third-order beam and changes the location of the optimum angle $\phi_{\mathrm{optm}}$.

\section{Uniformly Coated Parallel Slab}

To enhance the performance of the PS-DOAP of Fig. 1 substantially, we uniformly coat the top surface of the $\mathrm{SiO}_{2}$ slab with a transparent (single-layer or multilayer) interference thin film. A good choice for this film material is $\mathrm{ZnS}$, with a refractive index of 2.35 at $\lambda=633 \mathrm{~nm}$. For a film with a thickness of $d=70 \mathrm{~nm}$, Fig. 6 shows $R_{k}(k=0,1,2,3)$ as a function of $\phi_{0}$, and Fig. 7 shows $D_{N}$ as a function of $\phi_{0}$. Figure 6 indicates an improvement of $R_{3}$ in the range $0^{\circ}<\phi_{0}<70^{\circ}$. Boosting the power in the third-order beam is important for achieving a good signal-to-noise ratio in the fourth channel and avoiding a singularity. Figure 7 indicates that the performance of this new design is optimum at $\phi_{\text {optm }}=52^{\circ}$, where $D_{N}$ is maximum. Comparing Figs. 5 and 7 shows the advantage of a uniform coating on the top surface of the slab in permitting the operation of the PS-DOAP at lower angles.

\section{Optimization of the Coating Thickness}

We now determine the optimum film thickness $d$ that provides the largest powers for the second- and the third-order beams. Figure 8 gives the fractional powers in the second- and the third-order beams as functions of the thickness $d$ when $\phi_{0}$ is $45^{\circ}$ for a $\mathrm{ZnS}$ coating material. Figure 8 indicates that $R_{3}$ and $R_{4}$ are maximum when $d \cong 70 \mathrm{~nm}$, which is half of the film-thickness period at $45^{\circ}$.

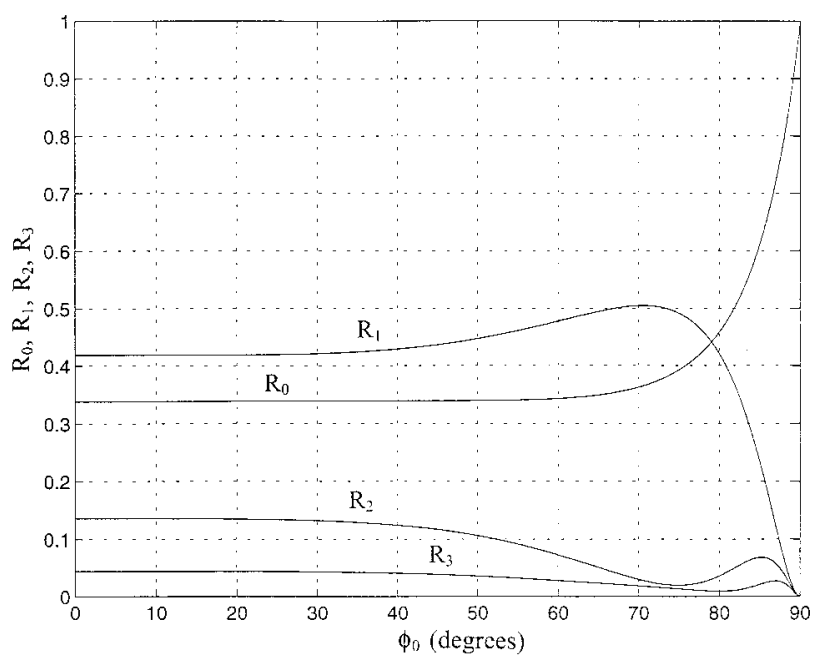

Fig. 6. Power reflectance $R_{k}(k=0,1,2,3)$ for the first four reflected orders as functions of the incidence angle $\phi_{0}$ obtained by use of a coated $\mathrm{ZnS}-\mathrm{SiO}_{2}-\mathrm{Ag}$ parallel slab at $\lambda=633 \mathrm{~nm}$. The thickness $d$ of the $\mathrm{ZnS}$ thin-film coating is $70 \mathrm{~nm}$.

\section{Optimization of the Angle of Incidence}

The choice of the optimum angle of incidence depends mainly on $R_{4}$ and the absolute value of $D_{N}$. Figures 6 and 7 suggest that $\phi_{\text {optm }}$ is in the range of $45^{\circ}$ to $50^{\circ}$. In Fig. $9 R_{4}$ is plotted as a function of $d$ when $\phi_{0}=$ $45^{\circ}, 47.5^{\circ}, 50^{\circ}$. Figure 9 shows that $R_{4}$ is largest when $\phi_{0}=45^{\circ}$ and $d=70 \mathrm{~nm}$. In Fig. $10 D_{N}$ is plotted as a function of $d$ when $\phi_{0}=45^{\circ}, 47.5^{\circ}, 50^{\circ}$. Figure 10 shows that $D_{N \max }$ occurs at $\phi_{\text {optm }}=50^{\circ}$. The difference of the normalized determinants at $\phi_{0}$ $=45^{\circ}, 50^{\circ}$ is less than $8 \%$, which has little effect on the singularity condition of the IM. Note that there is a trade-off between the optimum choices of $R_{4}$ and $D_{N}$ at the same angle. Near-optimum performance of this design is possible at $\phi_{\text {optm }}=45^{\circ}$.

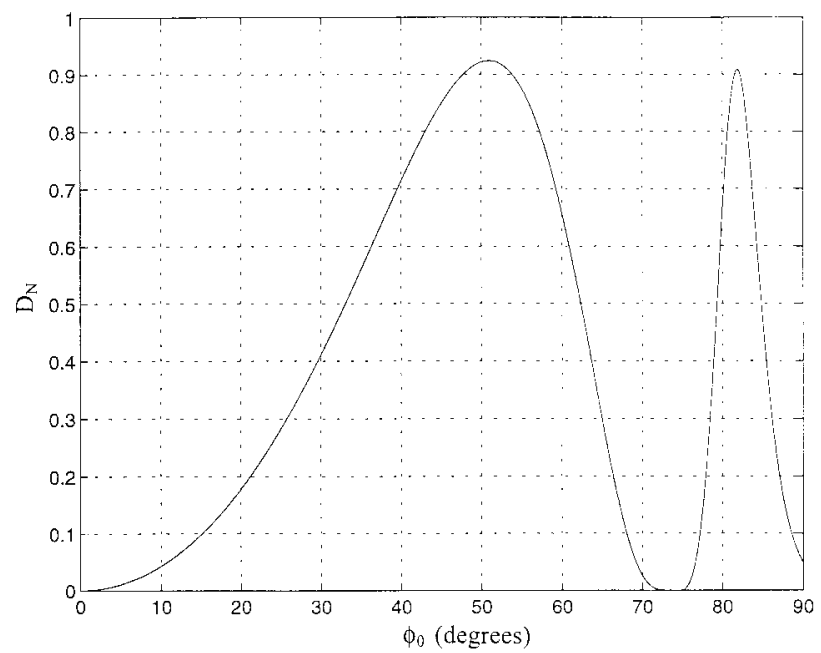

Fig. 7. Normalized determinant $D_{N}$ as a function of the incidence angle $\phi_{0}$ obtained by use of a coated $\mathrm{ZnS}-\mathrm{SiO}_{2}-\mathrm{Ag}$ parallel slab at $\lambda=633 \mathrm{~nm}$. The thickness $d$ of the $\mathrm{ZnS}$ thin-film coating is 70 $\mathrm{nm}$. 


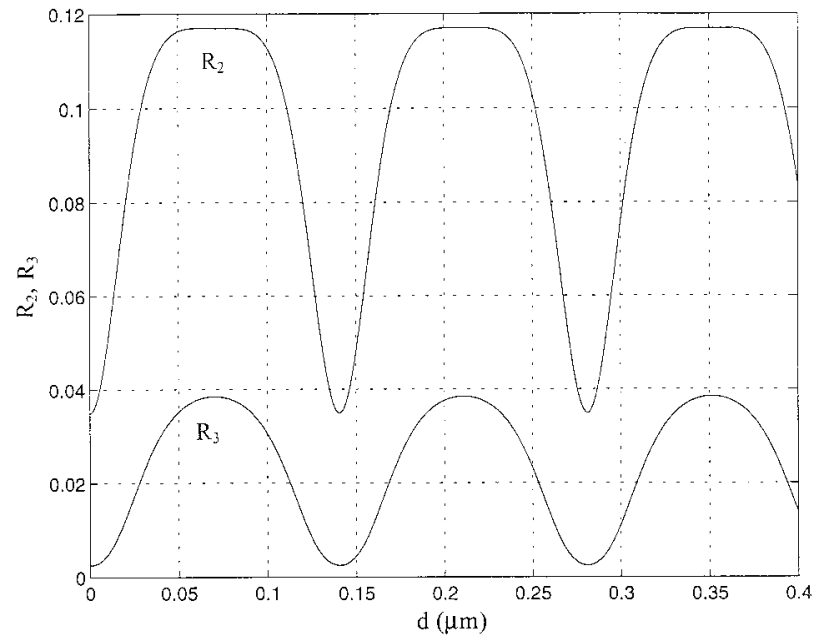

Fig. 8. Power reflectance $R_{k}(k=2,3)$ for the second and the third reflected orders as functions of the coating thickness $d$ obtained by use of a coated $\mathrm{ZnS}-\mathrm{SiO}_{2}-\mathrm{Ag}$ parallel slab at $\lambda=633 \mathrm{~nm}$ and an angle of incidence of $\phi_{0}=45^{\circ}$.

Another important parameter that affects the choice of the angle of incidence is the effect of lightbeam deviation (LBD) on the measurement of the input SOP by use of the PS-DOAP. This issue is considered in Section 7.

\section{Effect of Light-Beam Deviation on the Measured State of Polarization}

In this section we study the effect of LBD on the measured Stokes parameters, i.e., the errors introduced in the normalized Stokes parameters because of an error in $\phi_{0}$. We first examine the effect of LBD on a given linear input SOP (on the equator of a Poincaré sphere). We then consider general important states on the Poincaré sphere (elliptical SOP).

The PS-DOAP is assumed to have an IM $\mathbf{A}$ at $\phi_{0}$.

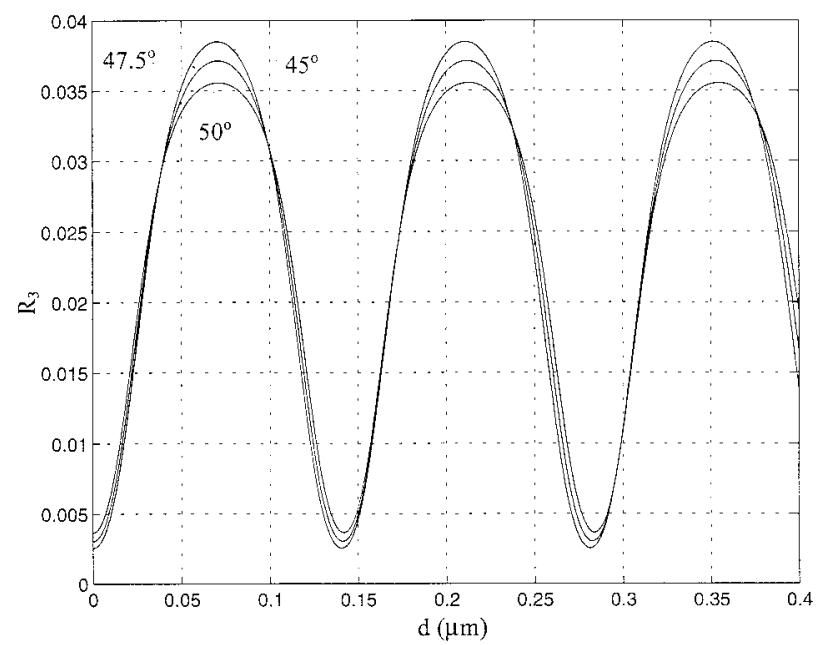

Fig. 9. Power reflectance $R_{k}(k=0,1,2,3)$ for the first four reflected orders as functions of the coating thickness $d$ obtained by use of a coated $\mathrm{ZnS}-\mathrm{SiO}_{2}-\mathrm{Ag}$ parallel slab at $\lambda=633 \mathrm{~nm}$. The angles of incidence are $\phi_{0}=45^{\circ}, 47.5^{\circ}, 50^{\circ}$.

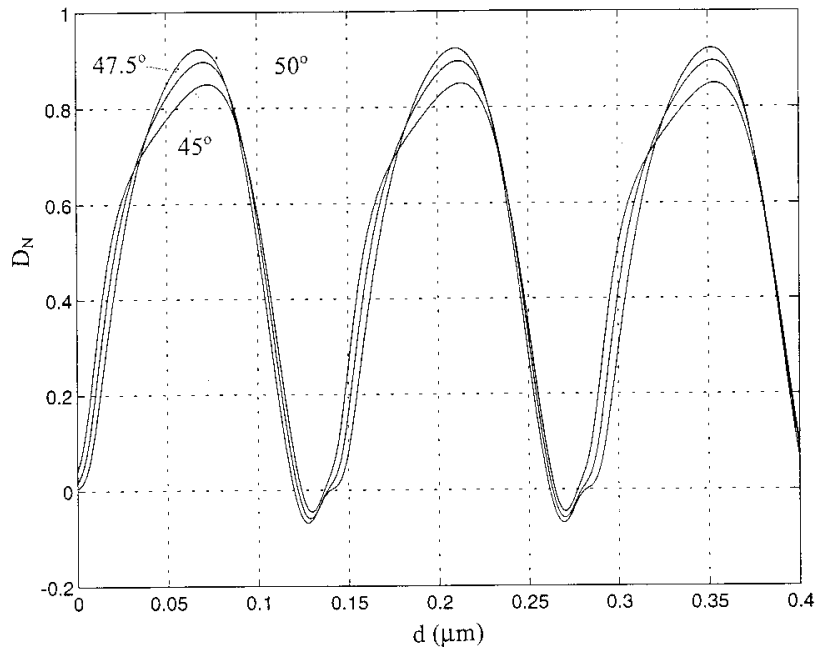

Fig. 10. Normalized determinant $D_{N}$ as a function of the coating thickness $d$ obtained by use of a coated $\mathrm{ZnS}-\mathrm{SiO}_{2}-\mathrm{Ag}$ parallel slab at $\lambda=633 \mathrm{~nm}$. The angles of incidence are $\phi_{0}=45^{\circ}, 47.5^{\circ}, 50^{\circ}$.

If an error $\Delta \phi_{0}$ is introduced in $\phi_{0}$ of, say, $0.5^{\circ}$, then the system's new IM A would be $\mathbf{A}^{\prime}$. In the presence of LBD, if $\mathbf{A}$ is used to measure the SOP $\mathbf{S}$ instead of $\mathbf{A}^{\prime}$ the measured SOP $\mathbf{S}^{\prime}$ is ${ }^{17}$

$$
\mathbf{S}^{\prime}=\mathbf{A}^{-1} \mathbf{A}^{\prime} \mathbf{S}
$$

The expression

$$
\Delta \mathbf{S}=\mathbf{S}-\mathbf{S}^{\prime}
$$

represents the error in the SOP $\mathbf{S}$ that is due to $\Delta \phi_{0}$. For our case the IM $\mathbf{A}$ is calculated to be

$$
\mathbf{A}=\left[\begin{array}{cccc}
0.4845 & -0.445 & 0.0000 & 0.0000 \\
0.4368 & 0.1808 & -0.3905 & 0.0750 \\
0.1143 & 0.1143 & 0.0000 & 0.0000 \\
0.0262 & 0.0099 & 0.0204 & -0.0132
\end{array}\right]
$$

To achieve equal values for the elements of the first column of Eq. (11), hence equal responses in the four detectors for incident unpolarized light, an electricalgain matrix $\mathbf{K}$ is introduced. ${ }^{13}$ In this case the gain matrix is

$$
\mathbf{K}=\left[\begin{array}{cccc}
1.0000 & 0.0000 & 0.0000 & 0.0000 \\
0.0000 & 1.1092 & 0.0000 & 0.0000 \\
0.0000 & 0.0000 & 4.2383 & 0.0000 \\
0.0000 & 0.0000 & 0.0000 & 18.4665
\end{array}\right],
$$

and the normalized IM A becomes

$$
\mathbf{A}=\left[\begin{array}{cccc}
0.4845 & -0.4845 & 0.000 & 0.0000 \\
0.4845 & 0.2005 & -0.4332 & 0.0832 \\
0.4845 & 0.4845 & 0.0000 & 0.0000 \\
0.4845 & 0.1826 & 0.3771 & -0.2434
\end{array}\right]
$$




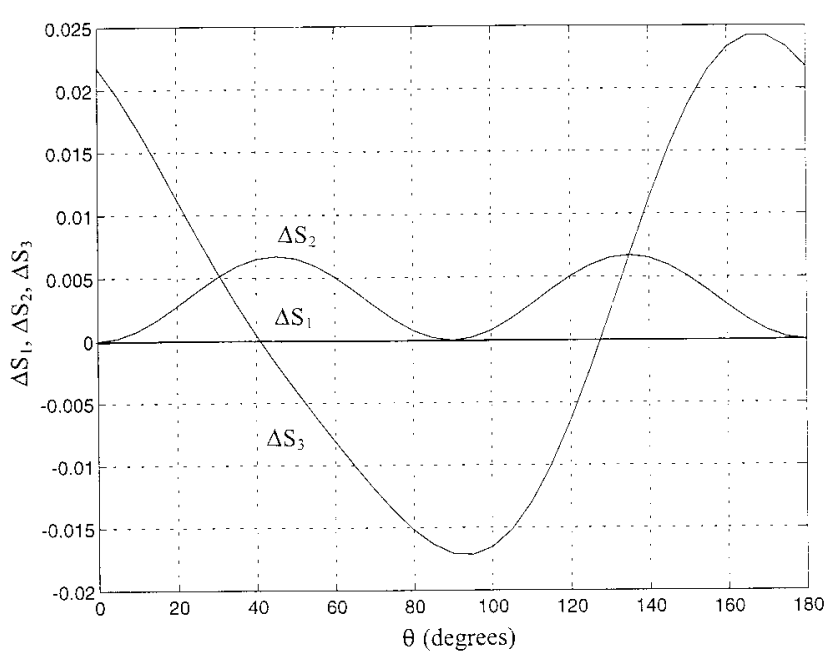

Fig. 11. Stokes parameters $\Delta S_{k}(k=1,2,3)$ as functions of the longitude angle $\theta$ obtained by used of a coated $\mathrm{ZnS}-\mathrm{SiO}_{2}-\mathrm{Ag}$ parallel slab at $\lambda=633 \mathrm{~nm}$ and an angle of incidence of $\phi_{0}=45^{\circ}$. The thickness of the $\mathrm{ZnS}$ thin-film coating is $70 \mathrm{~nm}$.

For $\phi_{0}=45.5^{\circ}\left(\right.$ hence $\Delta \phi_{0}=0.5^{\circ}$ ), Eq. (13) becomes

$$
\mathbf{A}=\left[\begin{array}{cccc}
0.4873 & -0.4873 & 0.000 & 0.0000 \\
0.4853 & 0.2043 & -0.4321 & 0.0842 \\
0.4808 & 0.4808 & 0.0000 & 0.0000 \\
0.4774 & 0.1751 & 0.3710 & -0.2440
\end{array}\right]
$$

The effect of the error $\Delta \phi_{0}$ on the measured input SOP is considered for a Poincaré sphere, where a point is represented by the latitude angle $2 \epsilon$ and the longitude angle 2 $\theta$. The input Stokes vector of a beam of light normalized to a unit intensity is given in terms of the ellipticity angle $\epsilon$ and the azimuth $\theta$ by ${ }^{15}$

$$
\mathbf{S}=\left[\begin{array}{c}
1 \\
\cos 2 \epsilon \cos 2 \theta \\
\cos 2 \epsilon \sin 2 \theta \\
\sin 2 \epsilon
\end{array}\right]
$$

Two cases of Eq. (14) are considered. First, the effect of LBD on the SOP is examined along the equator of a Poincaré sphere, hence the effect of LBD on all possible linear SOP's is determined. Second, we examine the effect of LBD on the elliptical SOP.

For the first case, we set $2 \epsilon=0$ in Eq. (14), which becomes

$$
\mathbf{S}=\left[\begin{array}{c}
1 \\
\cos 2 \theta \\
\sin 2 \theta \\
0
\end{array}\right]
$$

As $\theta$ sweeps $180^{\circ}, 2 \theta$ sweeps $360^{\circ}$ on the equator. Figure 11 shows the errors in the calculated normalized Stokes parameters plotted as functions of $\theta$ for $\Delta \phi_{0}=0.5^{\circ}$ at $\phi_{0}=45^{\circ}$ and $\lambda=633 \mathrm{~nm}$. Figure 11 indicates no error in the second Stokes parameter $\Delta S_{1}$ and small errors in the third and the fourth

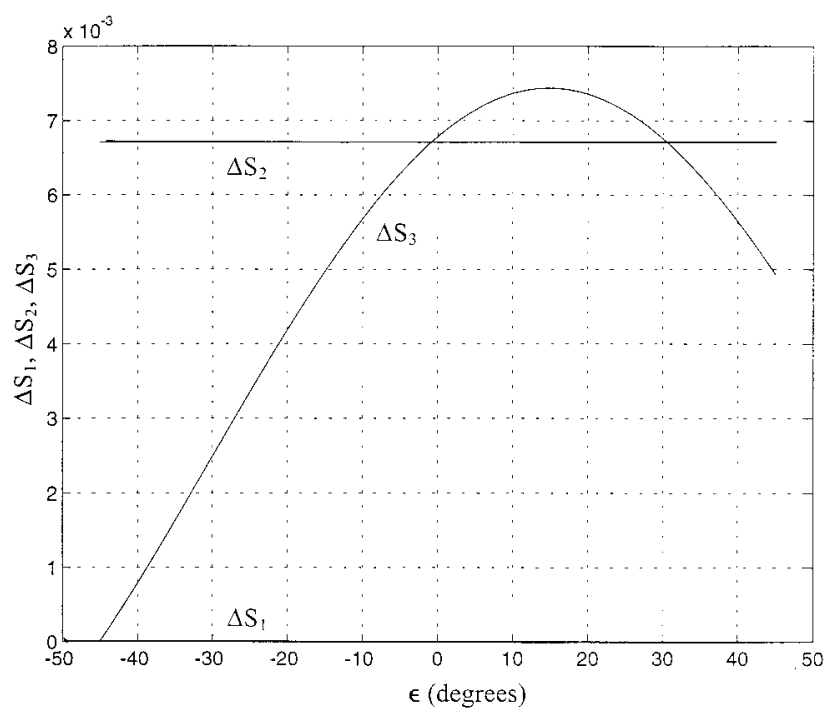

Fig. 12. Stokes parameters $\Delta S_{k}(k=1,2,3)$ as functions of the latitude angle $\epsilon$ obtained by used of a coated $\mathrm{ZnS}_{-} \mathrm{SiO}_{2}-\mathrm{Ag}$ parallel slab at $\lambda=633 \mathrm{~nm}$, an angle of incidence of $\phi_{0}=45^{\circ}$, and a longitude angle of $\theta=-45^{\circ}$. The thickness of the $\mathrm{ZnS}$ thin-film coating is $70 \mathrm{~nm}$.

Stokes parameters $\Delta S_{2}$ and $\Delta S_{3}$, respectively. The third Stokes parameter $\Delta S_{2}$ exhibits two equal maxima at $\theta=45^{\circ}$ and $\theta=135^{\circ}$. The first maximum error takes place when the input light is linearly polarized with an azimuth of $+45^{\circ}\left(L_{+45}\right)$ or is linearly polarized with an azimuth of $-45^{\circ}\left(L_{-45}\right)$. The maximum error in the fourth Stokes parameter $\Delta S_{3}$ takes place when $\theta=165^{\circ}$. The errors in the second and the third Stokes parameters are small with maximum values of $\left|\Delta S_{2}\right|<1 \%$ and $\left|\Delta S_{3}\right|<2.5 \%$, respectively, which are not excessive.

We now examine the effect of LBD on an input SOP represented by general points on a Poincaré sphere, i.e., the elliptical-polarization state. An elliptical SOP is represented by points on the Poincaré sphere excluding the south and the north poles and the equator. We let $\epsilon$ sweep $90^{\circ}\left(-45^{\circ}<\epsilon<45^{\circ}\right)$; hence $2 \epsilon$ sweeps a total of $180^{\circ}$ at four different longitudes of the Poincaré sphere: $\theta=-45^{\circ}, 0,+45^{\circ}, 90^{\circ}$. Figures 12-15 show plots of the errors in the normalized Stokes parameters as functions of the latitude angle $\epsilon$ at $\theta=-45^{\circ}, 0,+45^{\circ}, 90^{\circ}$, respectively, for $\Delta \phi_{0}=$ $0.5^{\circ}, \phi_{0}=45^{\circ}$, and $\lambda=633 \mathrm{~nm}$. These errors are small (i.e., of the order of $10^{-3}$ ). As before, the second Stokes parameters $\Delta S_{1}$ remains error free. From Fig. 13, we can see that the third Stokes parameter $\Delta S_{2}$ is negligible and the fourth Stokes parameter $\Delta S_{3}$ has a maximum of $2.1 \%$. Coordinates $(0,0)$ on the Poincaré sphere represent horizontal linear polarization, where $\Delta S_{1}=\Delta S_{2}=0$, according to Fig. 13. Figure 14 again shows that the value of the second Stokes parameter $\Delta S_{1}=0$ and that it is independent of LBD. From Fig. 14, note that the third Stokes parameter $\Delta S_{2}$ is constant over the entire range of $\epsilon$. Both the third and the fourth Stokes parameters $\Delta S_{2}$ and $\Delta S_{3}$ are negligible (maximum 


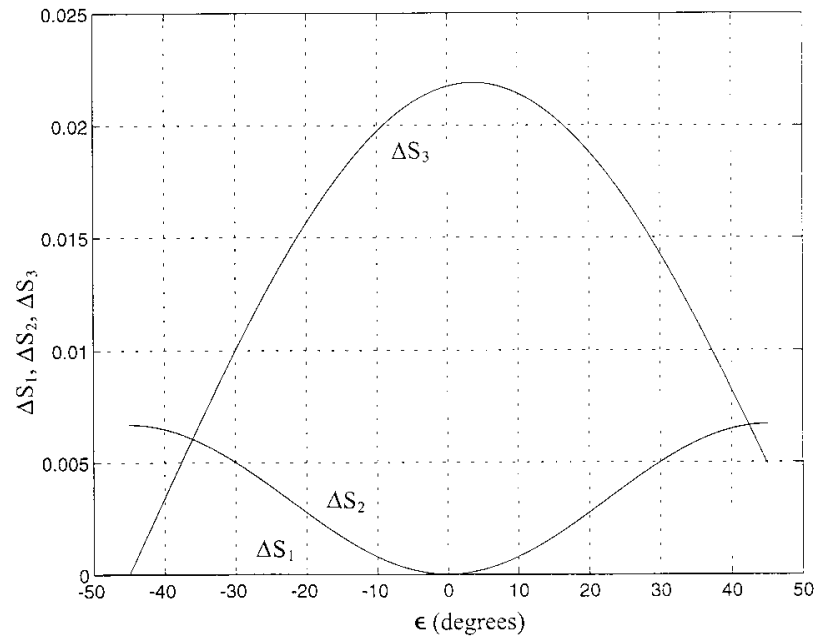

Fig. 13. Stokes parameters $\Delta S_{k}(k=1,2,3)$ as functions of the latitude angle $\epsilon$ obtained by use of a coated $\mathrm{ZnS}_{-} \mathrm{SiO}_{2}-\mathrm{Ag}$ parallel slab at $\lambda=633 \mathrm{~nm}$, an angle of incidence of $\phi_{0}=45^{\circ}$, and a longitude angle of $\theta=0$. The thickness of the $\mathrm{ZnS}$ thin-film coating is $70 \mathrm{~nm}$.

values less than $0.5 \%$ ) for this case. In Figure 15 similar observations can be made with respect to a point on the Poincaré sphere with coordinates of $\left(90^{\circ}, 0\right)$, which represents vertical linear polarization. The third Stokes parameter is $\Delta S_{2}=0$, and the fourth Stokes parameter $\Delta S_{3}$ approximately reaches its maximum at this point, whereas the second Stokes parameter $\Delta S_{1}$ is unchanged.

Finally, the dependence of LBD on $\phi_{0}$ is of interest. For values of $\phi_{0}<45^{\circ}$ the PS-DOAP is expected to have a lower sensitivity for a given LBD as long as the IM A remains nonsingular. Figure 16 plots the errors in the input normalized Stokes parameters as func-

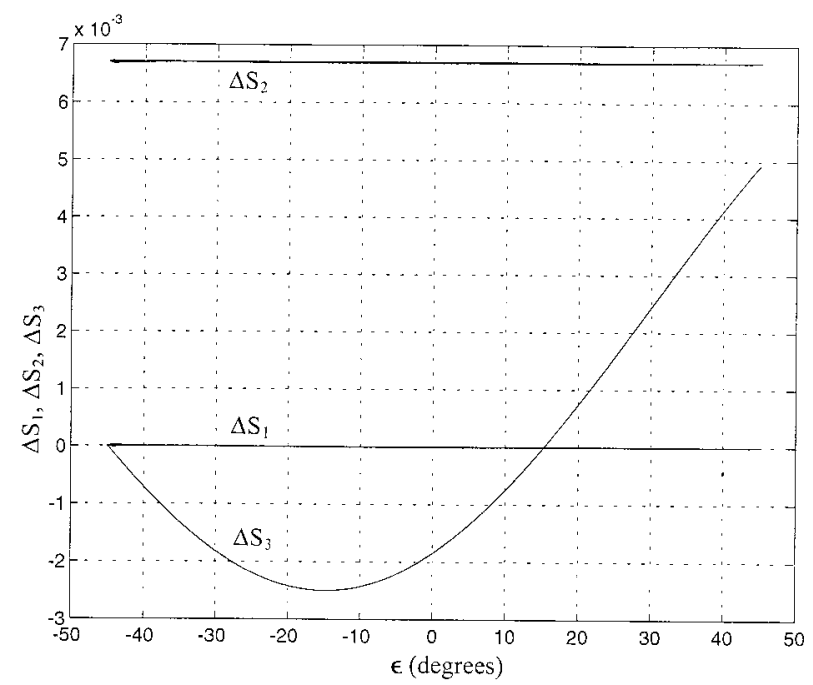

Fig. 14. Stokes parameters $\Delta S_{k}(k=1,2,3)$ as functions of the latitude angle $\epsilon$ obtained by use of a coated $\mathrm{ZnS}-\mathrm{SiO}_{2}-\mathrm{Ag}$ parallel slab at $\lambda=633 \mathrm{~nm}$, an angle of incidence of $\phi_{0}=45^{\circ}$, and a longitude angle of $\theta=45^{\circ}$. The thickness of the $\mathrm{ZnS}$ thin-film coating is $70 \mathrm{~nm}$.

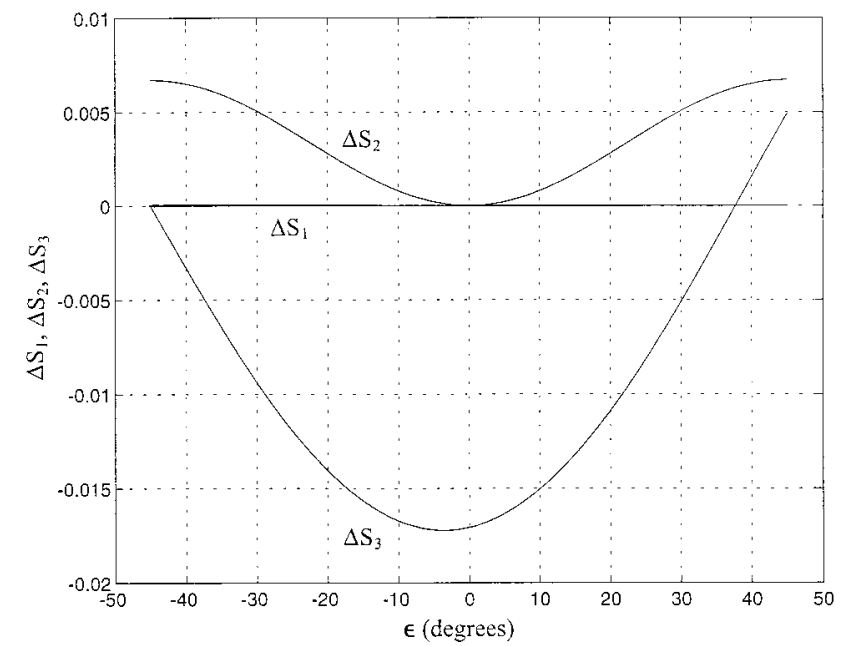

Fig. 15. Stokes parameters $\Delta S_{k}(k=1,2,3)$ as functions of the latitude angle $\epsilon$ obtained by use of a coated $\mathrm{ZnS}-\mathrm{SiO}_{2}-\mathrm{Ag}$ parallel slab at $\lambda=633 \mathrm{~nm}$, an angle of incidence of $\phi_{0}=45^{\circ}$, and a longitude angle of $\theta=90^{\circ}$. The thickness of the $\mathrm{ZnS}$ thin-film coating is $70 \mathrm{~nm}$.

tions of $\theta$ at $\phi_{0}=40^{\circ}$ and at $\phi_{0}=45^{\circ}$. It is evident from Fig. 16 that there are some improvements in the third $\Delta S_{2}$ and the fourth $\Delta S_{3}$ Stokes parameters when $\phi_{0}=40^{\circ}$. The first Stokes parameter $\Delta S_{2}$ is less by $0.2 \%$, whereas the second Stokes parameter $\Delta S_{3}$ is less by $20 \%$. We also note that, at $\phi_{0}=40^{\circ}, R_{3}$ remains nearly the same, whereas the normalized determinant $D_{N}$ decreases by $20 \%$. The normalized determinant $D_{N}$ remains far from zero, and a $20 \%$ reduction in the second Stokes parameter $\Delta S_{3}$ is obtained. Therefore a value of $\phi_{0}=40^{\circ}$ is recommended as a compromise optimum operating angle for this design.

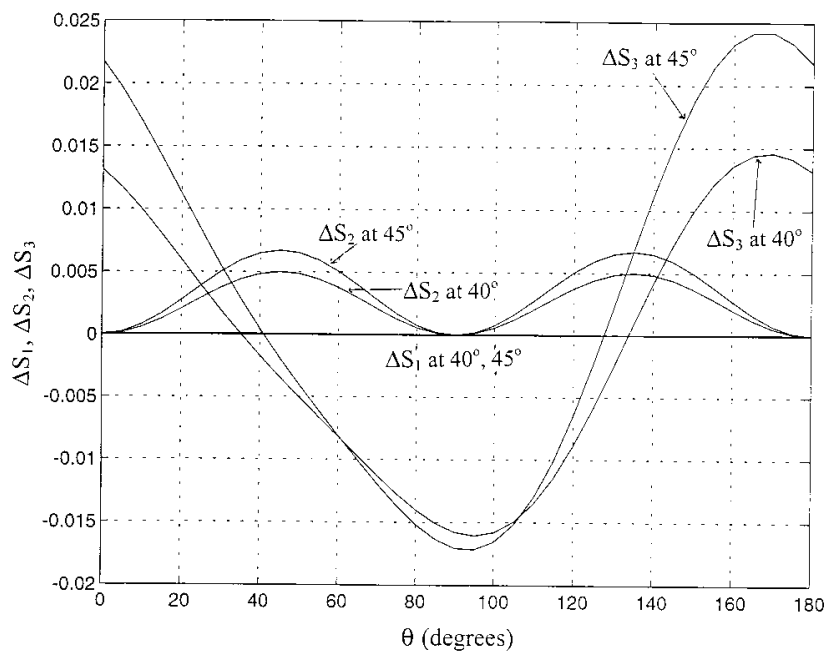

Fig. 16. Stokes parameters $\Delta S_{k}(k=1,2,3)$ as functions of the longitude angle $\theta$ obtained by use of a coated $\mathrm{ZnS}-\mathrm{SiO}_{2}-\mathrm{Ag}$ parallel slab at $\lambda=633 \mathrm{~nm}$ and angles of incidence of $\phi_{0}=40^{\circ}, 50^{\circ}$. The thickness of the $\mathrm{ZnS}$ thin-film coating is $70 \mathrm{~nm}$. 


\section{Conclusions}

Optimum conditions for operating a new DOAP that uses a coated dielectric-slab beam splitter have been determined. For a fused-silica slab an opaque $\mathrm{Ag}$ film on the back side and a 70-nm ZnS film on the front side yield a near-maximum normalized determinant of the IM at a $40^{\circ}$ angle of incidence and a 633-nm wavelength. At this general angle errors in the measured normalized Stokes parameters that are due to LBD are $<2 \%$ over the Poincaré sphere.

R. M. A. Azzam is currently on sabbatical with the Department of Physics, American University of Cairo, P.O. Box 2511, Cairo 11511, Egypt.

\section{References}

1. A. M. El-Saba, R. M. A. Azzam, and M. A. G. Abushagur, "Parallel-slab division-of-amplitude photopolarimeter," Opt. Lett. 21, 1709-1711 (1996).

2. R. M. A. Azzam, "Multichannel polarization state detectors for time-resolved ellipsometry," Thin Solid Films 234, 371-374 (1993).

3. R. M. A. Azzam and K. A. Giardina, "Polarization analysis based on grating conical diffraction," in Polarization Analysis and Measurement, D. H. Goldstein and R. A. Chipman, eds., Proc. SPIE 1746, 2-13 (1992).

4. R. M. A. Azzam, "Multidetector photopolarimeter for industrial optical sensing and metrology," in Industrial Applications of Optical Inspection, Metrology, and Sensing, G. M. Brown, K. G. Harding, and H. P. Stahl, eds., Proc. SPIE 1821, 270-283 (1993).

5. E. Collett, "Determination of the ellipsometric characteristics of optical surfaces using nanosecond laser pulses," Surf. Sci. 96, 156-167 (1980).
6. R. Cross, B. Heffner, and P. Hernday, "Polarization measurement goes automatic," Lasers and Optronics 10(11), 25-26 (1991).

7. A. S. Siddiqui, "Real time measuring of polarization," Photonics Spectra 26, 120-124 (1992).

8. R. M. A. Azzam, "Beam splitters for the division-ofamplitude photopolarimeter (DOAP)," Opt. Acta 32, 14071412 (1985).

9. K. Brudzewski, "Static Stokes ellipsometer: general analysis and optimization," J. Mod. Opt. 38, 889-896 (1991).

10. S. Krishnan, "Calibration, properties, and applications of the division-of-amplitude photopolarimeter at 632.8 and 1523 nm," J. Opt. Soc. Am. A 9, 1615-1622 (1992).

11. R. M. A. Azzam, "Recent developments of division-ofamplitude photopolarimeters," in International Symposium on Polarization Analysis and Applications to Device Technology, T. Yoshizawa and H. Yokota, eds., Proc. SPIE 2873, 1-4 (1996).

12. R. M. A. Azzam and K. A. Giardina, "Photopolarimeter based on a planar grating diffraction," J. Opt. Soc. Am A 10, 11901196 (1993).

13. R. M. A. Azzam, I. M. Elminyawi, and A. M. El-Saba, "General analysis and optimization of the four-detector photopolarimeter," J. Opt. Soc. Am. A 5, 681-689 (1988).

14. R. M. A. Azzam and A. G. Lopez, "Accurate calibration of the four-detector photopolarimeter with imperfect polarizing optical elements," J. Opt. Soc. Am. A 6, 1513-1521 (1989).

15. R. M. A. Azzam and N. M. Bashara, Ellipsometery and Polarized Light (North Holand, Amsterdam, 1977).

16. E. D. Palik, ed., Handbook of Optical Constants of Solids (Academic, New York, 1985).

17. J. Liu and R. M. A. Azzam, "Effect of light-beam deviation on the instrument matrix of the four-detector photopolarimeter," Opt. Eng. 36, 943-951 (1997). 